\title{
COVID-19 and Pregnancy: A Review
}

\author{
${ }^{1}$ Department of Obstetrics and Gynecology, All India Institute of \\ Medical Sciences, Jodhpur, Rajasthan, India \\ ${ }^{2}$ Department of Pediatrics, All India Institute of Medical Sciences, \\ Jodhpur, Rajasthan, India \\ ${ }^{3}$ All India Institute of Medical Sciences, Jodhpur, Rajasthan, India
}

Pratibha Singh $^{1}$ Manu Goyal ${ }^{1}$ Kuldeep Singh ${ }^{2}$ Sanjeev Misra ${ }^{3}$

\begin{abstract}
Address for correspondence Pratibha Singh, MBBS, MD, FMAS, Department of Obstetrics and Gynecology, All India Institute of Medical Sciences, Jodhpur 342005, Rajasthan, India (e-mail: drpratibha69@hotmail.com).
\end{abstract}

Ann Natl Acad Med Sci (India):2020;3:183-188

\begin{abstract}
Novel coronavirus disease 2019 (COVID-19) caused by severe acute respiratory syndrome-coronavirus-2 (SARS-CoV-2) has affected the lives of people across the globe, and Obstetricians are studying its effect on pregnancy and newborns. The pandemic resulted in major decisions taken by leaders in many countries, including strict policies as laying down of compulsory lockdown, curfews in high-risk places, and shut down of many services. This was done to promote physical distancing, thereby preventing community spread of the virus and for the preparedness of the health care facilities to deal with the pandemic. In the current scenario, the pregnant women who want to seek antenatal care are unable to visit the clinician and health center due to lockdown or for fear of contracting an infection. Reduced antenatal visits might lead to missing out some high-risk factors in due course of pregnancy and has the potential to adversely affect the health of women and newborn. Some key issues like optimizing antenatal and intranatal care with the prevailing situation, the effect of COVID-19 infection on fetus and newborn are addressed here. We searched PubMed (National Library of Medicine) and Embase (Elsevier) databases from January till July 2020, using a combination of the following keywords: "COVID-19," "SARS-CoV-2," and "pregnancy." Studies were selected if they met the following criteria: clinical studies, studies reporting original data, studies reporting SARS-CoV-2 infected women who were pregnant or had delivered. Here, we are providing the consensus made from the available literature. There is not much effect of COVID-19 infection on mother and fetus. The vertical

Keywords

- COVID-19 in pregnancy

- SARS-CoV-2 infection in pregnancy

- coronavirus infection in pregnancy transmission of the virus is also not yet proven. The timing and mode of delivery are not affected by the COVID-19 infection to the mother. Obstetric treatment should not be delayed for the COVID-19 testing or report. There are only a few studies reported for the management of pregnant women during pregnancy, based on which some principles formulated. However, more extensive studies are required to validate these principles. This review briefly describes the precautions needed to take during antenatal care.
\end{abstract}

\section{Introduction}

Novel coronavirus disease 2019 (COVID-19) first reported from Wuhan city of Hubei province, in China toward the end of the year 2019. It spreads rapidly to the nearby regions and soon engulfed many countries over a very short period of time. The outbreak was declared as public health emergency by the World Health Organization (WHO) in January 2020 and later global pandemic in March 2020 due to the highly infectious nature of the disease and the mortality related to it. The new coronavirus disease received an official name of COVID-19 in February 2020 by WHO., ${ }^{1,2}$ The pandemic 
resulted in major decisions taken by many countries, including strict policies as laying down of compulsory lockdown, curfews in high-risk places, and shut down of many services.

The etiological agent of severe acute respiratory syndrome-coronavirus-2 (SARS-CoV-2) is a single-stranded, nonsegmented, RNA enveloped virus belonging to the family coronaviridae and order nidovirales. These viruses also include SARS-CoV and Middle East respiratory syndromecoronavirus (MERS-CoV)., Since this is a new strain of coronavirus not seen previously in humans, very little is known about COVID-19 disease at present, particularly its effect on pregnant women and infants. The previous two related viruses (SARS and MERS) were associated with higher infectivity, severity, and mortality in pregnant women. ${ }^{5}$ Being a new virus, only a few studies related to coronavirus with pregnancy are there, and literature is evolving as new pieces of evidence are coming forward. Here, we provide a brief review of the clinical picture, pregnancy and fetal outcome, and vertical transmission of COVID-19 infection in pregnant women. We searched the literature on PubMed (National Library of Medicine) and Embase (Elsevier) databases from January till July 2020, using a combination of the following keywords: "COVID-19," "SARS-CoV-2," and "coronavirus" in pregnancy. Studies were selected if they met the following criteria: clinical studies, studies reporting original data, and studies reporting SARS-CoV-2 infected women who were pregnant or had delivered.

Prevention and treatment of COVID-19 infection among pregnant women and the likely risk of vertical transmission is a matter of major concern. Effective preventive and curative strategies are required practices to deal with this global pandemic. Long-term follow-up studies are needed to generate more evidence in this regard to direct the clinical practice. ${ }^{6,7}$

\section{Does the Rate of Pregnancy Complications Increase due to COVID-19 Infection?}

Previous studies showed a higher incidence of maternal adverse events like miscarriage, preterm labor, fetal growth restriction, and admission to the intensive care unit with SARS infection. ${ }^{8,9}$ But this anticipated increased risk is not seen in pregnant women with COVID-19 disease till now. Limited adverse events like abortion or stillbirth were reported with COVID-19, but it is not sure that these outcomes are directly related to maternal infection. ${ }^{6,7}$ The frequency of spontaneous abortions does not appear to be increased ${ }^{10,11}$; likewise, the rate of spontaneous preterm delivery expected to be similar to noninfected mothers. ${ }^{10}$ The results from various studies did not indicate any increased risk of perinatal complications in infected women, including the occurrence of severe preeclampsia, premature rupture of the membrane, fetal distress, meconium-stained amniotic fluid, premature delivery, neonatal asphyxia, and postpartum hemorrhage. ${ }^{10-13}$ Knight et al, in their study in the United Kingdom reported that more than half of women underwent cesarean delivery but majority done for obstetric indications rather than maternal compromise due to SARS-CoV-2 infection. ${ }^{14}$ In another study, there was an increase in iatrogenic preterm births and cesarean delivery rates to relieve respiratory distress by gravid uterus in SARS-CoV-2 infected mother. ${ }^{10}$ There are concerns regarding the psychosocial wellbeing of pregnant women and their families during the pandemic.

\section{The Course of COVID-19 Disease during Pregnancy}

Pregnant women are not more susceptible to COVID-19 infection than the general population. ${ }^{9,10}$ The disease presents with mild-to-moderate flu-like symptoms in the majority of women, and the clinical picture is almost similar in pregnant, as well as nonpregnant women. The rate of hospitalization in pregnant women is around $5 \%$, which is equivalent to that in nonpregnant one. ${ }^{10-14}$ The disease severity is more in immunocompromised females, and those having renal or cardiac disease, diabetes, or chronic illness. ${ }^{6}$ A recent systematic review shows that almost $75 \%$ of women presented in the third trimester. ${ }^{10}$ Less than one-fifth of women were asymptomatic, while most had a fever, cough, dyspnea, anosmia, and nonspecific complaints, including fatigue, myalgia, and headache. Obesity and comorbidities like diabetes, chronic hypertension in pregnant women are high-risk factors for the requirement of critical care, as in the general population..$^{10,13}$ Intensive care required in almost approximately $7 \%$ cases. ${ }^{10}$ A case fatality rate for U.K. pregnant women hospitalized with COVID-19 of $1.2 \%$ (95\% confidence interval [CI]: $0.4-2.7 \%$ ) and a SARS-CoV-2-associated maternal mortality rate of 5.6 (95\% CI: 1.8-13.1) per 100,000 maternities were reported. ${ }^{14,15}$

\section{Vertical Transmission}

The virus transmits from humans to humans mainly through droplets, aerosols, and fomites. Vertical transmission of the virus from pregnant women to fetuses is still controversial. Most of the large studies have shown swabs from the vagina, placental membranes, umbilical cord, amniotic fluid samples, cord blood, and breast milk samples were negative for Reverse transcription polymerase chain reaction (RT-PCR) for coronavirus infection. ${ }^{10-12}$ These studies have shown viral infection of the neonates during close contact with the mother or other hospital persons. However, few studies have raised the issue of possible vertical or intrauterine transmission of the disease due to positive SARS-CoV-2 in amniotic fluid and neonatal throat swab. ${ }^{16-18}$ Researchers have proposed two possible mechanisms for vertical transmission. One is through angiotensin-converting enzyme 2 (ACE2), which has been indicated recently as the putative surface receptor of sensitive cells for SARS-CoV-2; this receptor is expressed in the human placenta. Another way of transmission is damage in the placental barrier in severe maternal hypoxia due to SARS-CoV-2 infection. ${ }^{19}$ Prospective, more objective studies are needed to prove transmission in the womb in the future.

\section{Effect on the Fetus}

Till now, there is no documented adverse effect on fetus in terms of growth restriction, congenital malformations, congenital infection, or fetal death. The reason for this may be fewer women presenting with laboratory-confirmed COVID-19 in the first and second trimesters; most cases were in advanced gestation. There are no studies on cognitive 
abnormalities and neurodevelopment in neonates born to infected mothers; this requires both short- and long-term follow-up of infected mothers and babies.

\section{Antenatal Care}

There are specific issues during pregnancy, such as the timing of prenatal visits and screening tests in uninfected women and, in infected women, potential complications, mode of delivery, and care in the postnatal period. Pregnant women should continue visiting their consulting physician or maternity center if they do not belong to the hot-spot area or containment zones. ${ }^{20-22}$ Pregnant patients having a history of contact with a person with confirmed, probable, or suspected COVID-19 should self-isolate at home for 14 days and monitored for symptoms. Testing to be done if women become symptomatic. ${ }^{20}$

\section{Timing of Prenatal Visits}

There are guidelines in various countries to modify traditional protocols for prenatal visits. The American College of Obstetricians and Gynecologists (ACOG), the Society for Maternal-Fetal Medicine (SMFM), and Royal College of Obstetricians and Gynaecologists (RCOG) have issued guidance regarding prenatal care during the COVID-19 pandemic. ${ }^{20,23,24}$ These modifications include telemedicine consultation, reducing the number of hospital visits, and clubbing the antenatal tests in the same visit to minimize maternal contact with others. Most low-risk women should pay four to five visits in person to the consulting physician. The first visit planned at around 11 to 13 weeks of gestation, where women is checked thoroughly. She should be screened for any high-risk factor (preexisting comorbidities) at this visit and offered screening for aneuploidies by a combined test; further routine antenatal tests and counseling regarding nutrition done on the same visit. The second visit can be planned between 18 and 20 weeks of gestation when a detailed anomaly scan is done.

Further, women can consult on telemedicine, and later visits should be planned around 28 to 32 weeks to check fetal growth and maternal wellbeing. One visit may be planned near term at 36 to 37 weeks of gestation. On each visit, detailed history and clinical examination performed by the obstetrician and sonography ordered in cases of fetal growth disorders or maternal diabetes. The visits can be more frequent in high-risk women depending on the condition.

\section{Precautions during Antenatal Visits}

Most women attending maternity services are healthy and advised to maintain stringent social distancing. ${ }^{20,21}$ Besides following physical distancing, they should also wear a face mask while attending the clinic and in public places, and follow hygienic measures such as hand washing for a minimum of 20 seconds with an alcohol-based sanitizer. Women having flu-like symptoms should ideally inform the health facility telephonically and alert the hospital staff to take necessary measures. Health care workers should examine any women coming for antenatal checkup having suspicion or confirmation of COVID-19 after wearing full personal protective equipment (PPE), and the minimum persons should examine the patient in isolation facility. Staff should adhere to PPE guidelines and make every effort to observe social distancing measures at work, even when not in direct contact with patients. This includes handwashing, eating in designated areas, and maintaining a distance of $2 \mathrm{~m}$ between colleagues, where practical. ${ }^{21-23}$ Women who are self-isolating should defer antenatal visits for 14 days. ${ }^{20}$ Any women coming to a health care facility for pregnancy-related problems with or without symptoms of COVID-19 should receive immediate care, and care should not be delayed for SARS-CoV-2 testing. ${ }^{24}$

\section{Whom to Test for COVID-19}

The administrative agencies have made the testing policy, and followed as per national or regional guidelines. The availability of resources for testing and personnel should be taken into account whenever national policies formulated. All pregnant women need not be tested for the same. Symptomatic women or women with influenza-like illness or having travel history in the last 14 days, close contact with a positive person, having severe acute respiratory illness should be tested for COVID-19. This test is offered to women admitted to the hospital with one of the following:

- Clinical/radiological evidence of pneumonia.

- Acute respiratory distress syndrome (ARDS).

- Fever $\geq 37.8^{\circ} \mathrm{C}$.

AND at least one of following: acute, persistent cough, hoarseness, nasal discharge/congestion, shortness of breath, sore throat, wheezing, or sneezing.

Furthermore, it is recommended that women with an isolated fever should be investigated and treated according to the unit protocol. Investigations include sending a full blood count. If lymphopenia is identified on the complete blood count, testing for COVID-19 should also be offered. ${ }^{25,26}$

\section{Diagnosis of COVID-19}

Testing for COVID-19 infection is done as per regional guidelines. RT-PCR and Ig (immunoglobulin)-M antibody testing are done for diagnosis of COVID-19 infection. Throat swabs and nasopharyngeal swabs are taken from the suspected patients and tested for RT-PCR; blood samples also tested for antibodies. Tests should be done by the Centre for Disease Control and Prevention (CDC) recommended Kit following WHO guidelines for qRT-PCR. ${ }^{25,26}$ Sensitivity depends on the following factors: the specific RT-PCR assay, the type of specimen obtained (nasopharyngeal specimens have higher sensitivity than oropharyngeal or nasal specimens), the quality of the sample, and the duration of illness at the time of testing. Chest X-ray and computed tomography (CT) scan should not be withheld in pregnant women if indicated clinically for management and done using abdominal shielding.

\section{Management of COVID-19-Positive Women during Pregnancy}

Women with mild COVID symptoms without any obstetric problems can be managed at home with due instructions to reach the hospital in case of need. Moderate-to-severely 
symptomatic patients should be managed as an inpatient. A woman with moderate or severe COVID-19 symptoms who happens to be pregnant but with no immediate pregnancy issue should be cared for by the same multidisciplinary team as a nonpregnant woman with additional input from the maternity team. These women should be admitted to the isolation facility and not in the delivery area.

Pulse rate, temperature, blood pressure, oxygen saturation, and respiratory rate must be monitored in these women frequently. Cardiotocography (CTG) should monitor the fetal heart rate in the third trimester. Supportive care is recommended in the form of rest, restricted intravenous (IV) fluid management, oxygen supplementation by mask, and nutritional care. Antipyretics (paracetamol is preferred) and antibiotics for suspected bacterial infection may also be given. Hydroxychloroquine (HCQ) and chloroquine were initially used for moderate-to-severe symptoms, but later studies suggested no benefit from the administration of both of these drugs. ${ }^{27,28}$ Furthermore, maternal side effects in the form of abnormal heart rhythms and QT interval prolongation in electrocardiography are concerning. Many antiviral drugs are being evaluated for the treatment of COVID-19. Some of these agents are clinically available as a combination of lopinavir-ritonavir and newer drug, like remdesivir, for use in severely ill patients. ${ }^{29}$ The patient should be monitored closely for any sign of deterioration and should be shifted to intensive care unit (ICU) or on a ventilator if the patient fails to maintain oxygen saturation. For most women with COVID-19 and nonsevere illness who have no medical/obstetric indications for the prompt delivery, termination of pregnancy is not indicated. Severely ill patients between 32 to 34 weeks of gestation (or beyond) with COVID-19 pneumonia may benefit from early delivery. ${ }^{24,30}$ These patients in ICU should be considered for thromboprophylaxis with low molecular weight heparin (LMWH). ${ }^{31}$

\section{Care during Labor}

The onset of labor should be appropriately confirmed as per the standard of care in all women attending the delivery suite. Women who are suspect or confirmed cases of COVID-19 should be monitored in isolation room carefully for observations, including temperature, respiratory rate and oxygen saturation, uterine contractions, and progress of labor. ${ }^{20}$ Fetal monitoring should be done by electronic fetal monitoring (EFM) using CTG. ${ }^{30}$ Efforts made to minimize the number of staff members entering the room, and units should develop a local policy specifying essential personnel for emergency scenarios. Asymptomatic birth partners asked to wash their hands frequently. If symptomatic, birth partners should remain in self-isolation at home for 2 weeks and not attend the unit. The neonatal team should be given sufficient notice at the time of birth, to allow them time to wear PPE before entering the delivery room/theater. ${ }^{22,23}$ Some people were using early cord clamping to minimize the time of exposure in COVID-19-positive women but delayed cord clamping still recommended following birth, provided there are no other contraindications. The baby can be cleaned and dried as usual, while the cord is still intact. ${ }^{20,23}$

\section{Mode of Delivery}

There is currently no evidence to favor vaginal or cesarean delivery in SARS-CoV-2 infected women. Therefore, it should be discussed with the patient, taking into consideration her preferences, and any obstetric indications for intervention. The presence of COVID-19 should not influence the mode of delivery unless the woman's respiratory condition demands urgent intervention for birth. The cesarean section usually reserved for obstetric indications. However, it may be considered to expedite delivery in critically ill patients in maternal and fetal interest. Delivery should be conducted with PPE and all precautions by health care workers. ${ }^{22}$ Women admitted for delivery with confirmed COVID-19 should be given thromboprophylaxis with LMWH unless contraindicated. ${ }^{20,30,31}$ It is given as injection enoxaparin $40 \mathrm{mg}$ subcutaneously once daily until 2 weeks after delivery.

\section{Breastfeeding}

Breastfeeding is continued irrespective of COVID-19 status of mother as benefits of breastmilk outweigh the risk due to COVID-19. Breast milk is a passive source of antibodies and has other protective factors. Most breastmilk samples in studies done till now had come negative for this virus, which suggests that this virus is not transmitted through milk. ${ }^{7,10}$ It is recommended that a mother who is a confirmed or suspected case of COVID-19 should take all precautions to avoid spreading the infection to the infant. She should follow breast and hand hygiene and wear a face mask while breastfeeding. COVID-19 positive mothers who want to breastfeed may feed expressed breast milk using proper breast and hand hygiene or directly breastfeed their infants wearing a mask while practicing proper breast and hand hygiene. ${ }^{22,32}$

There is no consensus for breast feeding; the WHO recommends breastfeeding initiation within an hour of birth and routine newborn care ${ }^{32}$; while current AAP (American Academy of Pediatrics) guidelines recommends rooming-in and to continue breast feeding, the previous guidance recommended physical separation from their infant when space allows, unless they choose despite being counseled on risk. ${ }^{33}$ Temporary separation of suspected or known COVID-19 positive mothers from a newborn is advocated to reduce the risk of mother-baby transmission. Still, it may also have adverse consequences, such as not rooming-in and avoiding skin-to-skin contact, that can be stressful for mothers, disrupt breastfeeding, and have negative effects on newborn feeding and bonding. WHO has opined that mothers who have suspected, probable, or confirmed COVID-19 virus infection should be enabled to remain together with their infants and practice skinto-skin contact. ${ }^{32}$ The CDC advises determining whether to separate a mother with known or suspected COVID-19 and her infant on a case-to-case basis, using shared decision making between the mother and the clinical team. ${ }^{34}$ Because of the paucity of data, separation of infants may seem the safest approach, but one must weigh the potential benefits of isolation against the real costs, for both women and infants, of losing postpartum contact. 


\section{Psychosocial Impact}

COVID-19 outbreak has resulted in increased anxiety among pregnant women because of concerns regarding the wellbeing of an unborn child, and aggravated by quarantine policy, physical distancing, home isolation, remote consultation with a health care professional, and inability to obtain the expected level of support and care in the antenatal and postpartum period. Uncertainties regarding the effect on fetal health, miscarriage, and preterm labor have added to psychological stress. This stress has an adverse impact on mental health, leading to fear, anger, and confusion. ${ }^{35}$ It might take time to have enough evidence that pregnant women are at high risk for depression, anxiety, and posttraumatic disorder in this pandemic. ${ }^{36}$ Hence, all women should be appropriately counseled both in the antenatal and postnatal period about the wellbeing of their baby, and they should be given tender loving care. It is prudent to recommend continued support from the family and health care provider, physically or by phone in the postpartum period.

\section{Conclusion}

COVID-19 pandemic has posed a great challenge for health care workers, as well as for pregnant women. Although the course of pregnancy is not much affected by COVID-19 disease itself, complications may arise in the presence of comorbidities. The absence of timely care and caution can lead to adverse events. Both the expecting mother and care provider should be vigilant, and the routine obstetric management should not be delayed due to testing or reports of COVID-19 infection. Generally, the management of labor is not altered in women giving birth during the COVID-19 pandemic or in women with confirmed or suspected COVID-19 disease. COVID-19 is not an indication to modify the route of delivery. Mother to child transmission is not proven yet but may occur. As the pandemic is still progressively involving many continents and people across the globe, we may have further data and clarifications in the near future on aspects not yet answered.

\section{Conflict of Interest}

None declared.

\section{References}

1 World Health Organization. Coronavirus dis- ease (COVID-19) pandemic. Available at: https://www.who.int/emergencies/ diseases/novel-coronavirus-2019. Accessed July 18, 2020

2 Rasmussen SA, Smulian JC, Lednicky JA, Wen TS, Jamieson DJ. Coronavirus disease 2019 (COVID-19) and pregnancy: what obstetricians need to know. Am J Obstet Gynecol 2020;222(5):415-426

3 Zhu N, Zhang D, Wang W, et al; China Novel Coronavirus Investigating and Research Team. A novel coronavirus from patients with pneumonia in China, 2019. N Engl J Med 2020;382(8):727-733

4 Huang C, Wang Y, Li X, et al. Clinical features of patients infected with 2019 novel coronavirus in Wuhan, China. Lancet 2020;395(10223):497-506
5 Wong SF, Chow KM, Leung TN, et al. Pregnancy and perinatal outcomes of women with severe acute respiratory syndrome. Am J Obstet Gynecol 2004;191(1):292-297

6 Qiao J. What are the risks of COVID-19 infection in pregnant women? Lancet 2020;395(10226):760-762

7 Chen H, Guo J, Wang C, et al. Clinical characteristics and intrauterine vertical transmission potential of COVID-19 infection in nine pregnant women: a retrospective review of medical records. Lancet 2020;395(10226):809-815

8 Lam CM, Wong SF, Leung TN, et al. A case-controlled study comparing clinical course and outcomes of pregnant and non-pregnant women with severe acute respiratory syndrome. BJOG 2004;111(8):771-774

9 Chen N, Zhou M, Dong X, et al. Epidemiological and clinical characteristics of 99 cases of 2019 novel coronavirus pneumonia in Wuhan, China: a descriptive study. Lancet 2020;395(10223):507-513

10 Khalil A, Kalawat E, Benlioglu C, et al. SARS-CoV-2 infection in pregnancy: A systematic review and meta-analysis of clinical features and pregnancy outcomes. E-Clin Med 2020. Doi: $10.1016 /$ j.eclinm.2020.100446

11 Yan J, Guo J, Fan C, et al. Coronavirus disease 2019 (COVID-19) in pregnant women: A report based on 116 cases. Am J Obstet Gynecol 2020;223(1):111.e1-111.e14

12 Liu Y, Chen H, Tang K, Guo Y. Clinical manifestations and outcome of SARS-CoV-2 infection during pregnancy. J Infect 2020. Doi: 10.1016/j.jinf.2020.02.028

13 Mullins E, Evans D, Viner R, et al. Coronavirus in pregnancy and delivery: rapid review. Ultrasound Obstet Gynaecol 2020; 55(5):-586-592

14 Knight M, Bunch K, Vousden N, et al; UK Obstetric Surveillance System SARS-CoV-2 Infection in Pregnancy Collaborative Group. Characteristics and outcomes of pregnant women admitted to hospital with confirmed SARS-CoV-2 infection in UK: national population based cohort study. BMJ 2020;369:m2107

15 Docherty AB, Harrison EM, Green CA, et al; ISARIC4C investigators. Features of 20133 UK patients in hospital with covid-19 using the ISARIC WHO Clinical Characterisation Protocol: prospective observational cohort study. BMJ 2020;369:m1985

16 Alzamora MC, Paredes T, Caceres D. Webb CM, Valdez LM, La Rosa M. Severe COVID-19 during pregnancy and possible vertical transmission. Am J Perinatol 2020;37(8):861-865

17 Zamaniyan M, Ebadi A, Aghajanpoor Mir S, Rahmani Z, Haghshenas M, Azizi S. Preterm delivery, maternal death, and vertical transmission in a pregnant woman with COVID-19 infection. Prenat Diagn 2020. Doi: 10.1002/pd.5713

18 Dong L, Tian J, He S, et al. P1ossible vertical transmission of SARS-CoV-2 from an infected mother to her newborn. JAMA 2020;323:1846-1848

19 Juan J, Gil MM, Rong Z, Zhang Y, Yang H, Poon LC. Effect of coronavirus disease 2019 (COVID-19) on maternal, perinatal and neonatal outcome: systematic review. Ultrasound Obstet Gynecol 2020;56(1):15-27

20 Royal college of Obstetricians \& Gynecologists (RCOG). Coronavirus 2019 (COVID-19) Infection in pregnancy: information for healthcare professionals. Version 9, Published 13, May 2020. Available at: https://www.rcm.org.uk/media/4059/202005-13-coronavirus-covid-19-infection-in-pregnancy.pdf?dm $\mathrm{i}=4 \mathrm{YCH}, \mathrm{C9JC}, 3 \mathrm{PNKLQ}, 1 \mathrm{DE} 66,1$. Accessed August 16, 2020

21 UK Government. COVID-19: guidance on social distancing and for vulnerable people. Available at: https://www.gov.uk/government/publications/covid-19-guidance-on-social-distancing-and-for-vulnerablepeople. Accessed July 15, 2020

22 Chen D, Yang H, Cao Y, et al. Expert consensus for managing pregnant women and neonates born to mothers with 
suspected or confirmed novel coronavirus (COVID-19) infection. Int J Gynaecol Obstet 2020;149(2):130-136

23 American college of Obstetricians \& Gynecologists. ACOG practice advisory on COVID-19 and obstetric care. Available at: https://www.linksmedicus.com/news/acog-practice-advisory-covid-19-obstetric-care/. Accessed August 4, 2020

24 Boelig RC, Saccone G, Bellussi F, Berghella V. MFM guidance for COVID-19. Am J Obstet Gynecol MFM 2020;2(2):100106

25 World Health Organization. Laboratory testing for 2019 novel coronavirus (2019-nCoV) in suspected human cases. Available at: https://www.who.int/publications-detail/laboratory-testing-for-2019-novel-coronavirus-in-suspected-human-cases-20200117. Accessed July 12, 2020

26 Corman VM, Landt O, Kaiser M, et al. Detection of 2019 novel coronavirus (2019-nCoV) by real-time RT-PCR. Euro Surveill 2020;25(3):2000045

27 Gautret P, Lagier JC, Parola P, et al. Hydroxychloroquine and azithromycin as a treatment of COVID-19: results of an open-label non-randomized clinical trial. Int J Antimicrob Agents 2020;56(1):105949

28 Anuvi R. COVID-19 in pregnancy- review of guidelines in indian setting from the point of view of community health. Int J Sci Healthcare Res 2020;5(2):138-143

29 Wang M, Cao R, Zhang L, et al. Remdesivir and chloroquine effectively inhibit the recently emerged novel coronavirus (2019-nCoV) in vitro. Cell Res 2020;30(3):269-271

30 López M, Gonce A, Meler E, et al; on behalf of the COVID Collaborative Group. Coronavirus disease 2019 in pregnancy: a clinical management protocol and considerations for practice. Fetal Diagn Ther 2020;47(7):519-528

31 American Society of Hematology. COVID-19 and VTE/anticoagulation: frequently asked questions. Available at: https:// www.hematology.org/covid-19/covid-19-and-vte-anticoagulation. Accessed July 20, 2020

32 World Health Organization. Breastfeeding and COVID-19. Available at: https://www.who.int/publications/i/item/ 10665332639. Accessed July 20, 2020

33 American Academy of Pediatrics. FAQs: Management of infants born to mothers with suspected or confirmed COVID1.9 Available at: https://services.aap.org/en/pages/2019-novel-coronavirus-covid-19-infections/clinical-guidance/ faqs-management-of-infants-born-to-covid-19-mothers/. Accessed July 22, 2020

34 Centers for Disease control and prevention. Considerations for inpatient obstetric healthcare settings. Available at: https:// www.cdc.gov/coronavirus/2019-ncov/hcp/inpatient-obstetric-healthcare-guidance.html. Accessed April 09, 2020

35 Brooks SK, Webster RK, Smith LE, et al. The psychological impact of quarantine and how to reduce it: rapid review of the evidence. Lancet 2020;395(10227):912-920

36 Thapa SB, Mainali A, Schwank SE, Acharya G. Maternal mental health in the time of the COVID-19 pandemic. Acta Obstet Gynecol Scand 2020;99(7):817-818 